\title{
Interactive 3D Time-Integrated Solar Shadow Maps
}

\author{
Georg Molzer ${ }^{\mathrm{a}^{*}}$, Florian Ledermann ${ }^{\mathrm{a}}$ \\ ${ }^{\mathrm{a}}$ Research Group Cartography, TU Wien, Vienna, Austria \\ *corresponding author,georg@molzer.org
}

Keywords: Interactive Map, 3D Cartography, Sun, Shadow, Solar shadows, WebGL, CityGML

Urban areas are characterized by a complex topography of buildings, terrain, vegetation and temporary structures, which, depending on their extent, geometry, geographic location and daytime, cast shadow on their surroundings. Given the importance of sunlight for various groups of interest and tasks, we argue that a comprehensive, accessible, usable and intuitive way of predicting its availability for specific usage contexts is surprisingly lacking. In our research, we are investigating how to improve the visual communication of urban solar conditions for various real-world usage scenarios like having a coffee in the sun, parking a car in the shade, or taking a photograph of a particular building in a favorable light. All of these activities take place over a period of time, not in a temporal instant, causing solar shadows to move. Hence, a static representation of the light situation at a distinct point in time, such as offered by available 3D urban maps or GIS systems, is often not sufficient for planning above mentioned recreational or professional activities.

This factor is incorporated in our system by integrating shadow motion into a shadow map that covers arbitrary timeframes within a given day (Figure 1). Shadows are accumulated by projecting shadow maps from astronomically precise sun positions spanning over the defined timeframe. The number of sampling points directly influences the quality and speed of the rendering. All provided figures currently sample and integrate nine different sun positions.

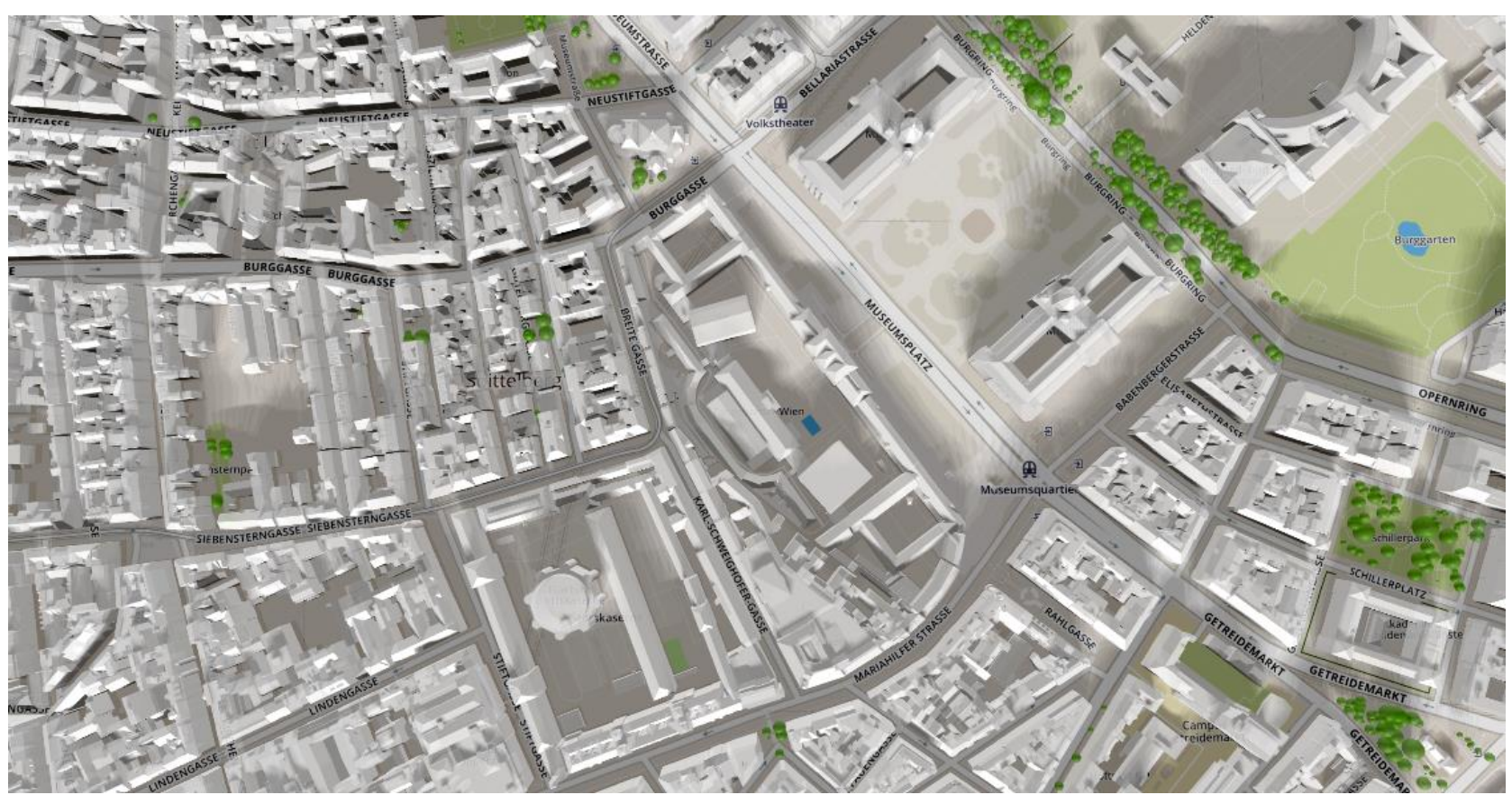

Figure 1. Vienna's 7th district on January 8th, with shadows aggregated between 11 am and 1pm local time. Even though the sun is at its highest position, most streets lie in at least partial, often in complete shadow. However, some parks and squares are fully lit over the entire timeframe.

Using our system, questions in a manner of "Given a specific point in space, at which time will this point be in sun/shade again" can be answered. E.g., one would like to spend time in the sun at a specific Viennese "Kaffeehaus" (coffee place), from which time on (and for how long) will this space be sunlit (Figure 2). In the future, this process might even be automated. 
The geometries required for accurately calculating the lighting situation in an urban environment need to be combined from different data sources, in different formats, often in different coordinate reference systems. This in turn requires a powerful and scalable pipeline to incorporate all relevant aspects to render solar shadows, with the eventual aim of creating a flexible system that will be working globally and for any city that provides 3D models of its buildings and other potentially relevant layers of information. A web-based interactive map is currently being built that combines 3D terrain data, 3D building data, tree cadasters and a basemap. Our prototype system is based on WebGL (via the Three.js Javascript 3D-engine), allowing realtime-interaction in any modern web browser.

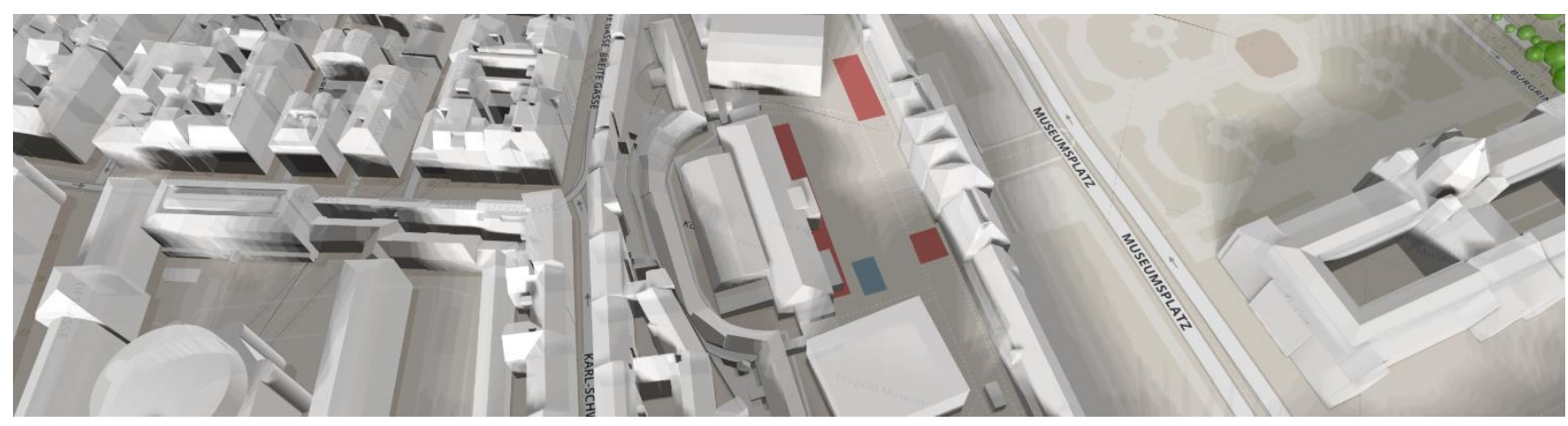

Figure 2. Vienna's "Museumsquartier" (center of image) is a favorite location for tourists and locals to enjoy art and relax in the sun. The visualization takes place at the same day and time period as Figure 1, restaurants with outside tables are highlighted red. The tilt of the viewpoint reveals strong shadowing on building facades, indicating that even the upper floors of higher buildings often do not receive direct sunlight due to sun's low altitude in winter.

The map is draggable and zoomable, dynamically loading required resources, covering the whole earth. Its basemap and terrain are streamed from tileservers. Terrain elevation is based on RGB-encoded height values that - decoded provide a vertical resolution of $10 \mathrm{~cm}$. A vertex shader displaces 3D-planes accordingly. After this process, our terrain is also able to cast and receive shadows, further contributing to an encompassing and realistic rendering.

As an initial case study, the city of Vienna has been selected, as it is widely known for its open approach towards government data. There is a CityGML (LOD2) dataset of buildings freely available. Via conversion and lossy 3D model compression, we could reduce its file size by $99.95 \%$ down to $47 \mathrm{MB}$. Vienna's tree cadaster on the other hand is a massive single >100 MB JSON file, covering metadata and location of 199.826 trees, requiring prior processing and tiling to become computable. While these optimizations are desirable in general, they become crucial in a web-context: The often redundant, heterogenous data sources need to be compressed and minimized in order to reduce loading times and also integrated in a way performant enough to allow 3D real time web-based visualization.

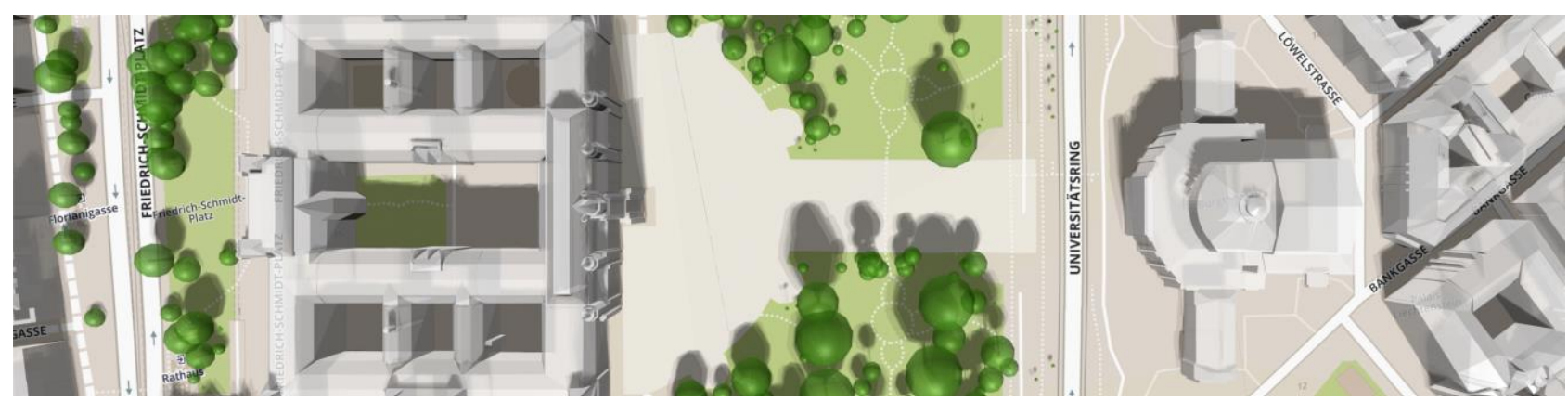

Figure 3. Vienna, July 8th, 2019, 12:00-12:30: If the weather allows, one of the city's most sun exposed times of the year as the sun is close to its maximum altitude. Vienna's Mayor can easily flee the sun in the townhall's inner yard (building on the left, constructed after Vienna's publicly available LOD 2 CityGML/DXF models) while passers-by can relax under a tree (location, size and diameter based on Vienna's publicly available tree catastre) in the townhall's park.

We expect to apply learnings from Vienna to other cities and to further automate data preprocessing and pipeline improvements, such as the integration of weather forecasts for short-term queries, in the future. 